\title{
The Estimation Method of 1/f Noise Parameters Based on Wavelet and Power Spectrum Estimation
}

\author{
Mao $\mathrm{Li}^{1+}$, Guofu Liu ${ }^{1}$ and Zicheng Guo ${ }^{1}$ \\ ${ }^{1}$ Department of Instrument Science and Technology, National University of Defense Technology, Changsha \\ 410073, China;
}

\begin{abstract}
. 1/f noise is closely related to the defects and damage inside the semiconductor device, and its noise parameters can characterize the reliability and performance of semiconductor devices. The basic characteristics of $1 / \mathrm{f}$ noise, the basic theory of wavelet transform and the basic principle of power spectrum estimation are introduced in this paper. The noise simulation test system based on the embedded development platform is designed and built, and the parameters of 1/f noise are estimated by the method based on the wavelet and power spectrum estimation. The results show that the method based on wavelet and power spectrum estimation has high accuracy and is suitable for $1 / \mathrm{f}$ noise parameter estimation under white noise background.
\end{abstract}

Keywords: 1/f noise, wavelet, power spectrum, parameters estimation, semiconductor device.

\section{Introduction}

$1 / \mathrm{f}$ noise is a low frequency noise which is commonly found in semiconductor devices. A large amount of useful information can be extracted from the data obtained from the 1/f noise to characterize the reliability of the electronic device $[1,2]$, so it is important to analyze the $1 / \mathrm{f}$ noise to obtain the noise parameters.

$1 / \mathrm{f}$ noise is a non-stationary random process. The power spectral density of the $1 / \mathrm{f}$ noise sequence $s(t)$ in the semiconductor device can be expressed by the following mathematical equation [3]

$$
S_{s}(f)=\frac{\sigma_{s}^{2}}{f^{\gamma}}
$$

where $f$ is the frequency; $\gamma$ is the frequency index factor of $1 / \mathrm{f}$ noise; $\sigma_{s}^{2}$ is the $1 / \mathrm{f}$ noise amplitude. It can be seen from Eq.(1) that the power spectral density of 1/f noise has a characteristic of increasing with increased noise amplitude and decreased frequency.

In recent years, many experts and scholars have spent a lot of effort to study many aspects of $1 / \mathrm{f}$ noise [4, 5]. In the aspect of parameter estimation of $1 / \mathrm{f}$ noise, the method based on power spectrum estimation is widely used [6], and the method based on wavelet has also been proposed [7]. However, both methods have some shortcomings. In order to improve the performance of the parameter estimation, we consider combining this two methods and their advantages, which is described in detail below.

\section{Estimation of 1/f Noise Parameters}

\subsection{Method based on power spectrum estimation}

The low frequency noise in semiconductor devices is usually composed of $1 / \mathrm{f}$ noise, white noise and g-r noise. It is difficult to observe g-r noise in large-size semiconductor devices because of the great modern

\footnotetext{
+ Corresponding author. Tel.: 18390900049

E-mail address: limao1993@foxmail.com.
} 
fabrication process. Therefore, this paper only considers $1 /$ f noise $s(t)$ and white noise $w(t)$, and the power spectral density can be written as

$$
S(f)=S_{s}(f)+S_{w}(f)=\frac{\sigma_{s}^{2}}{f^{\gamma}}+\sigma_{w}^{2}
$$

where $\sigma_{w}^{2}$ is the white noise amplitude. When the frequency tends to infinity, the following equation can be obtained

$$
\lim _{f \rightarrow \infty} S(f)=\lim _{f \rightarrow \infty}\left(\frac{\sigma_{s}^{2}}{f^{\gamma}}+\sigma_{w}^{2}\right)=\sigma_{w}^{2}
$$

In order to convert Eq.(2) into a linear expression, we can subtract $\sigma_{w}^{2}$ and take a logarithm on both sides of Eq.(2), then the following equation can be obtained

$$
\lg \left(S(f)-\sigma_{w}^{2}\right)=-\gamma \lg f+\lg \left(\sigma_{s}^{2}\right)
$$

At this point, $\lg f$ and $\lg \left(S(f)-\sigma_{w}^{2}\right)$ are treated as observed data, $\gamma$ and $\sigma_{s}^{2}$ are treated as unknown parameters. For Eq.(4), we can use the least squares estimation method to obtain $\gamma$ and $\sigma_{s}^{2}$.

The method based on power spectrum estimation is simple and fast, but there are some shortcomings. First, the accuracy of this estimation method depends on the magnitude of the error of the power spectrum calculation method and the smoothness of the spectral line. In addition, Eq.(2)-(4) convert the nonlinear relationship into a linear relationship. It can be seen from Fig.1 that the data points are non-uniformly distributed in the double logarithmic coordinate system. This actually makes the weight of the lower frequency part smaller than the weight of the higher frequency part, and the original data of equal uncertainty become data of unequal uncertainty, which will have a large impact on the accuracy of the estimation.

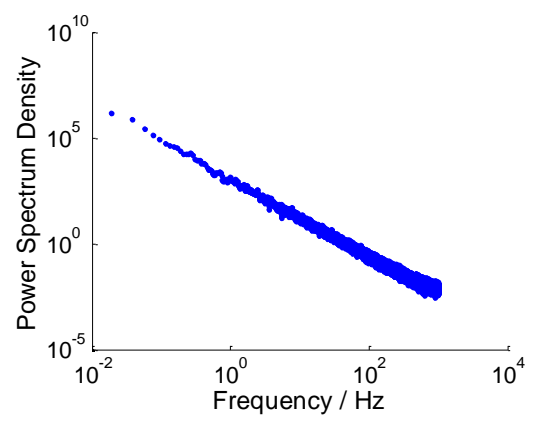

Fig. 1: The power spectrum estimation of $1 / \mathrm{f}$ noise.

\subsection{Method based on wavelet}

For dyadic wavelet, it can be expressed as $[8,9]$

$$
\psi_{j k}(t)=2^{-j / 2} \psi\left(2^{-j} t-k\right)
$$

where $\psi(t)$ is the wavelet basis; $j$ is the scale factor; $k$ is the time displacement variable. For the $1 / f$ noise under white noise background

$$
x(t)=s(t)+w(t)
$$

the dyadic wavelet transform coefficients $s_{k}^{j}$ of 1/f noise $s(t)$ can be expressed as

$$
s_{k}^{j}=W T_{s}(j, k)=\int s(t) \psi_{j k}(t) \mathrm{d} t=2^{-j / 2} \int s(t) \psi\left(2^{-j} t-k\right) \mathrm{d} t
$$

And the dyadic wavelet transform coefficients $w_{k}^{j}$ of white noise $w(t)$ can be expressed as

$$
w_{k}^{j}=W T_{w}(j, k)=\int w(t) \psi_{j k}(t) \mathrm{d} t=2^{-j / 2} \int w(t) \psi\left(2^{-j} t-k\right) \mathrm{d} t
$$

The dyadic wavelet transform coefficients $x_{k}^{j}$ of $x(t)$ can be written as

$$
x_{k}^{j}=s_{k}^{j}+w_{k}^{j}
$$

The variance of can be proved as [10]

$$
\operatorname{var}\left(x_{k}^{j}\right)=2^{\gamma j} \sigma_{1}^{2}+\sigma_{2}^{2}
$$


where $\sigma_{1}^{2}$ is a constant proportional to $\sigma_{s}^{2}$ and $\sigma_{2}^{2}$ is a constant proportional to $\sigma_{w}^{2}$. Therefore, the variance of the wavelet coefficients with the scale $j+1$ can be written as

$$
\operatorname{var}\left(x_{k}^{j+1}\right)=2^{\gamma(j+1)} \sigma_{1}^{2}+\sigma_{2}^{2}
$$

Subtracting Eq.(10) by Eq.(11), and taking the logarithm with the bottom of 2, the following equation can be obtained

$$
\log _{2}\left\lfloor\operatorname{var}\left(x_{k}^{j+1}\right)-\operatorname{var}\left(x_{k}^{j}\right)\right\rfloor=\gamma j+\log _{2}\left\lfloor\left(2^{\gamma}-1\right) \sigma_{1}^{2}\right\rfloor
$$

At this point, $j$ and $\log _{2}\left[\operatorname{var}\left(x_{k}^{j+1}\right)-\operatorname{var}\left(x_{k}^{j}\right)\right]$ are treated as observed data, $\gamma$ and $\log _{2}\left[\left(2^{\gamma}-1\right) \sigma_{1}^{2}\right]$ are treated as unknown parameters. For Eq.(4), we use the least squares estimation method to obtain $\gamma$.

In practical application, compared with the method of power spectrum estimation, the wavelet analysis method has higher accuracy for $\gamma$, but the estimation of $\sigma_{s}^{2}$ is more complicated. Therefore, we consider combining the power spectrum estimation method with the wavelet method. We use wavelet analysis to estimate $\gamma$, and then use estimated $\gamma$ as a known parameter and use power spectrum estimation method to estimate $\sigma_{s}^{2}$.

\section{Experiment and Simulation}

\subsection{Equipment and solutions of experiment}

The simulation and detection system of 1/f noise was built in this paper. As shown in Fig.2, the entire 1/f noise simulation testing system mainly consists of ARM processor, FPGA, ADC, DAC, and PC.

The sequence of 1/f noise was generated by the ARM processor and transferred into the FPGA, and then converted into analog signals by the DAC. The signal was sampled by the ADC and transferred into the PC by the FPGA. The sampled data was lastly analyzed and processed in the PC.

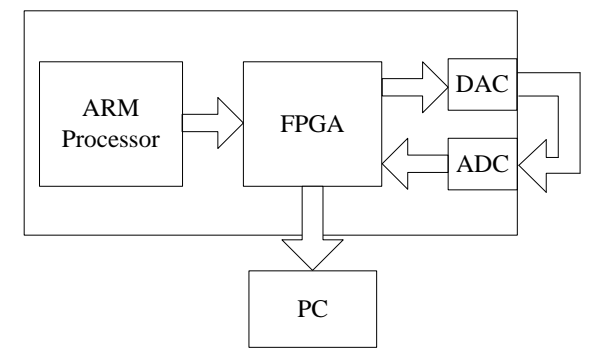

Fig. 2: The simulation and detection system of $1 / \mathrm{f}$ noise.

\subsection{Results and analysis of experiment}

According to the method of [11], 1/f noise was generated. In this experiment, 1/f noise parameter $\gamma$ was set to 1.8 and $\sigma_{s}^{2}$ was set to 1000 . ADC sampling frequency was $f_{\mathrm{s}}=10 \mathrm{kHz}$, the number of samples was $N_{\mathrm{s}}=2^{22}$.

In this paper, with the consideration of accuracy and calculation speed, the Welch spectrum estimation method is used in the spectral estimation, and the Haar wavelet is used in the wavelet analysis, which is analyzed by repetitive experiments.

The white noise is added to the generated 1/f noise, and the time domain waveform is shown in Fig.3. When the SNR (signal to noise ratio) is less than $5 \mathrm{~dB}, 1 / \mathrm{f}$ noise is submerged in the additive white noise.

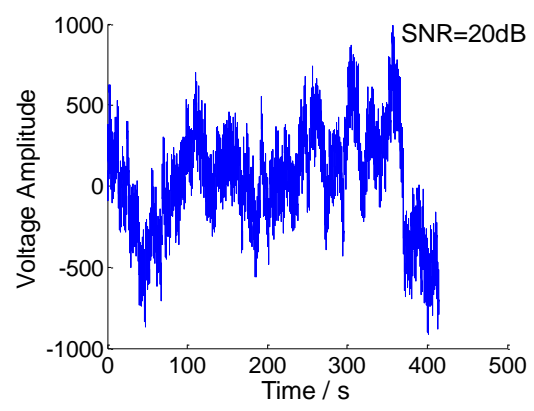

(a)

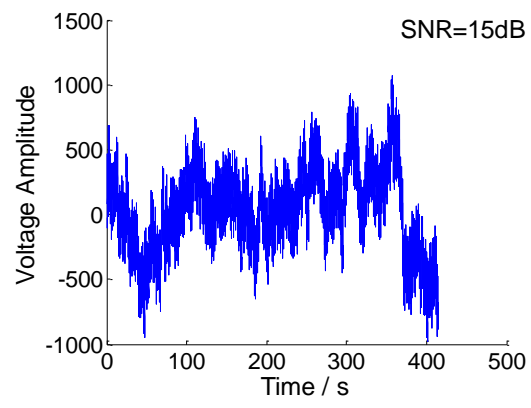

(b)

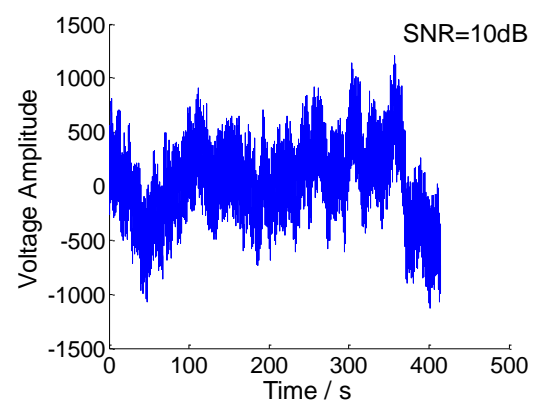

(c) 


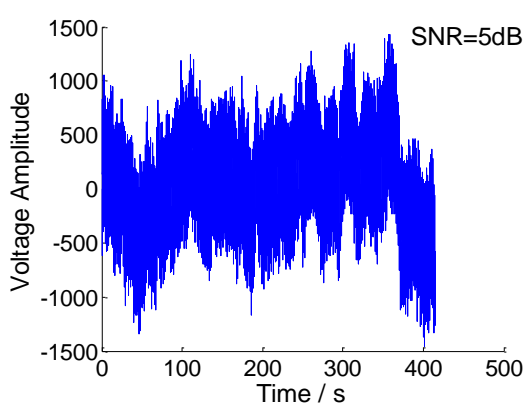

(d)

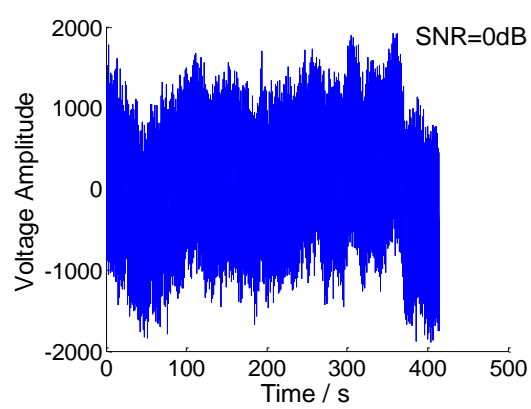

(e)

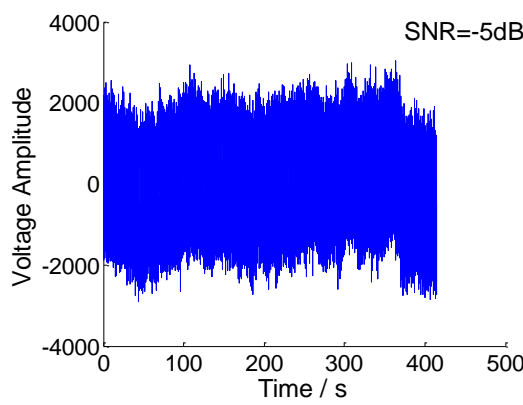

(f)

Fig. 3: Waveform of 1/f noise in different SNR. (a) SNR=20dB; (b) SNR=15dB; (c) SNR=10dB; (d) SNR=5dB; (e) $\mathrm{SNR}=0 \mathrm{~dB}$; (f) $\mathrm{SNR}=-5 \mathrm{~dB}$.

According to Eq.(12), the value $\gamma$ of $1 / \mathrm{f}$ noise is estimated by wavelet analysis and least squares method. The results are shown in Fig.4.

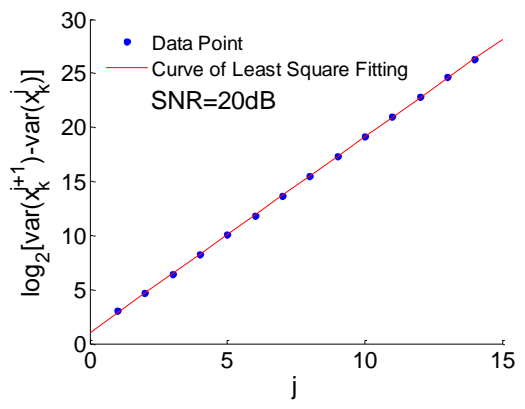

(a)

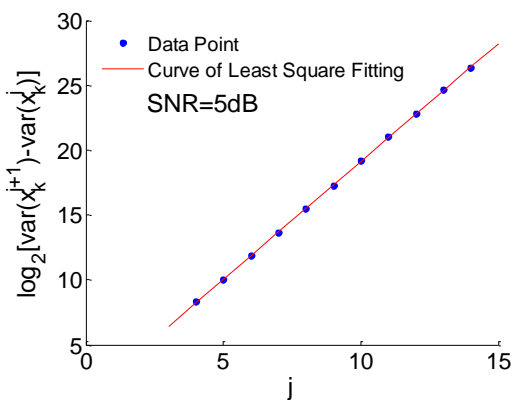

(d)

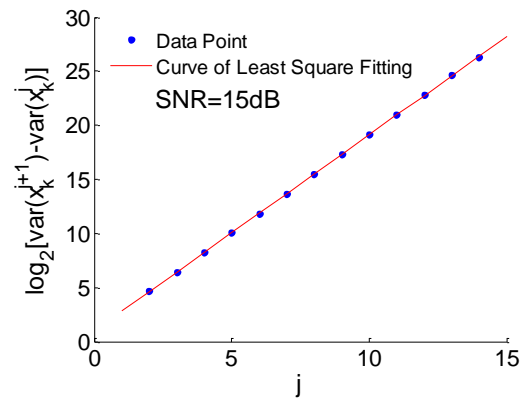

(b)

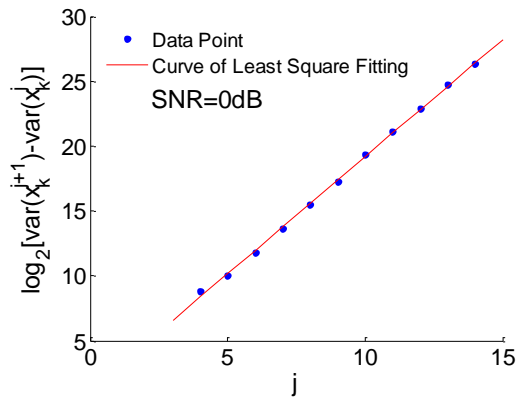

(e)

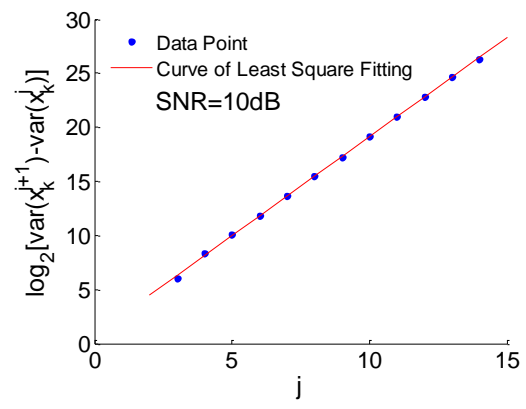

(c)

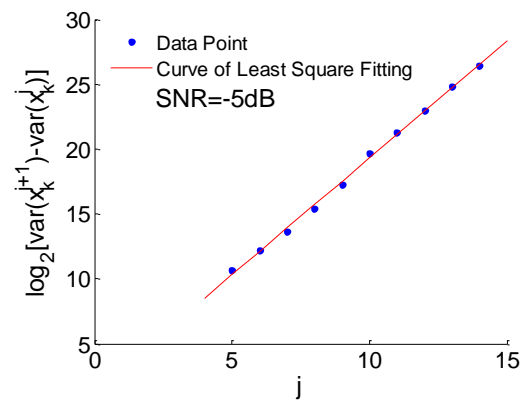

(f)

Fig. 4: Estimation of $\gamma$ in different SNR. (a) $\hat{\gamma}=1.7970$; (b) $\hat{\gamma}=1.7927$; (c) $\hat{\gamma}=1.8093$; (d) $\hat{\gamma}=1.8129$; (e)

$$
\hat{\gamma}=1.8177 \text {; (f) } \hat{\gamma}=1.8254 \text {. }
$$

The power spectrum of 1/f noise is calculated by the Welch method. According to Eq.(4), the estimated $\gamma$ is used as the known parameter and the $\hat{\sigma}_{s}^{2}$ can be estimated by the least squares method. The results are shown in Fig.5.

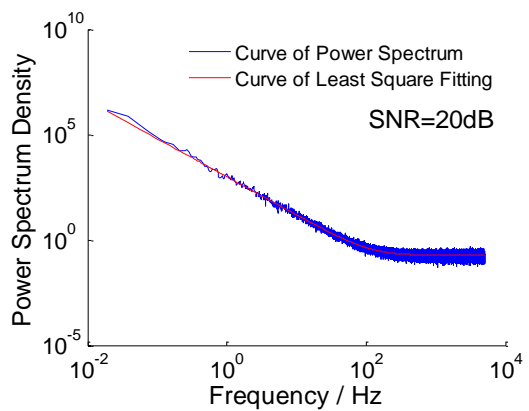

(a)

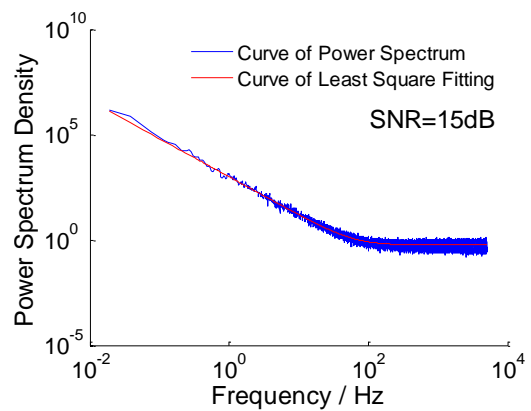

(b)

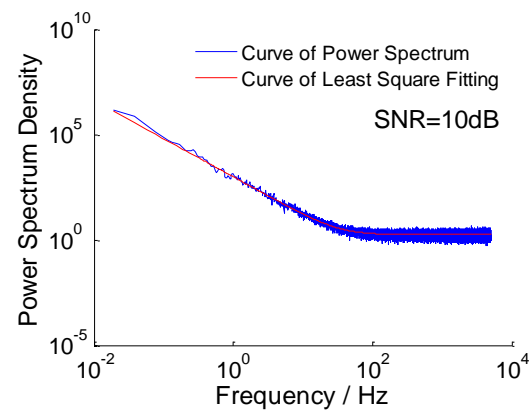

(c) 


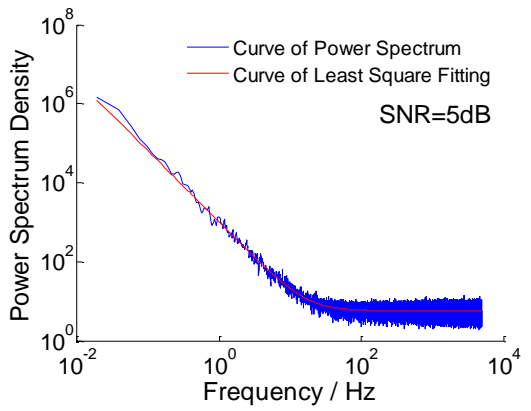

(d)

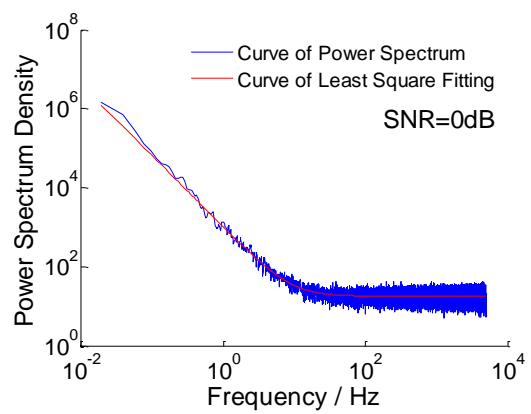

(e)

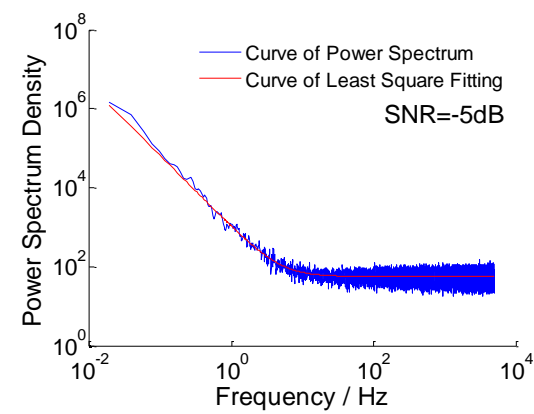

(f)

Fig. 5: Estimation of $\sigma_{s}^{2}$ in different SNR. (a) $\sigma_{s}^{2}=996.1431$; (b) $\sigma_{s}^{2}=1007.7354$; (c) $\sigma_{s}^{2}=1013.7866$; (d)

$$
\sigma_{s}^{2}=983.6539 ; \text { (e) } \sigma_{s}^{2}=1021.8555 ; \text { (f) } \sigma_{s}^{2}=971.8378 \text {. }
$$

From Fig.4 and Fig.5, the relative error of the estimation result can be calculated, as shown in Fig.6. With the increasing of SNR from $-5 \mathrm{~dB}$, the estimation error of $\gamma$ and $\sigma_{s}^{2}$ is getting smaller and smaller, which is within the acceptable range. However, when SNR is less than $-10 \mathrm{~dB}$, it can also be found that the linear relationship between $\log _{2}\left[\operatorname{var}\left(x_{k}^{j+1}\right)-\operatorname{var}\left(x_{k}^{j}\right)\right]$ and $j$ become weaker, which makes the estimation error larger than 5\%. Therefore, the $1 / \mathrm{f}$ noise in the background of the white noise can be moderately denoised to improve the estimation performance in the practical application.

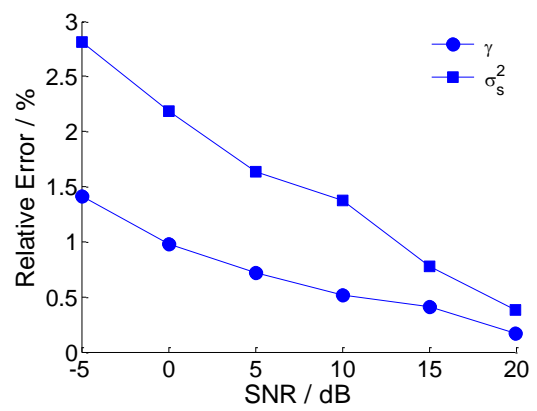

Fig. 6: The relative error of estimation result.

\section{Summary}

In this paper, the shortcomings of spectral estimation and wavelet analysis for parameters estimation of 1/f noise are analyzed. The relationship between the power spectral density and the frequency and the relationship between wavelet transform coefficient and scale are constructed, so that the parameters of 1/f noise can be estimated by the method based on wavelet and power spectrum estimation. And the process of $1 / \mathrm{f}$ noise parameter estimation based on this method is described in detail. From the experimental results, the method of estimating $1 / \mathrm{f}$ noise parameters in the background of white noise is of high performance and feasibility.

\section{Acknowledgements}

This project was financially supported by the National Natural Science Foundation of China under Grant no. 11375264.

\section{References}

[1] Vandamme L K J. Noise as a diagnostic tool for quality and reliability of electronic devices. IEEE Transactions on Electron Devices, 1994, 41(11):2176-2187.

[2] Zhuang Yiqi, Sun Qing. Noise as tool to characterize electron device reliability. Acta Electronica Sinica, 1996(2):76-82(in Chinese).

[3] Bonani F, Ghione G. Noise in semiconductor devices. Modeling and Simulation. Springer, 2001.

[4] Chen Haipeng, Cao Junsheng, Guo Shuxu. Temperature-dependent relation between junction temperature and 1/f noise in high power semiconductor laser. Acta Phys. Sin., 2013, 62(10):197-202. 
[5] Lakehal M R, Ferdi Y, Taleb-Ahmed A. On the self-similarity of 1/f $\beta$ sequences synthesized by recursive filtering. Computers \& Electrical Engineering, 2012, 38(2):282-293.

[6] G. Giusi, G. Scandurra, C. Ciofi. Estimation errors in 1/f $\gamma$ noise spectra when employing DFT spectrum analyzers. Fluctuation \& Noise Letters, 2013, 12(01).

[7] Ran Peng, Guo Shuxu, Cheng Xiaogou. The parameter test and estimation of 1/f noise. Journal of Test \&Measurement Technology, 2013, 27(1): 46-49.

[8] Chui C K, Jiang Q. Wavelet Transform and Filter Banks. Applied Mathematics. Atlantis Press, 2013.

[9] Bose N K. Wavelets and filter banks. Applied Multidimensional Systems Theory. Springer International Publishing, 2017.

[10] Wornell G W, Oppenheim A V. Estimation of fractal signals from noisy measurements using wavelets. IEEE Transactions on Signal Processing, 1992, 40(3):611-623.

[11] Wornell G W, Oppenheim A V. Wavelet-based representations for a class of self-similar signals with application to fractal modulation. IEEE Transactions on Information Theory, 1992, 38(2):785-800. 